\title{
IMPROVING IMAGE MATCHING BY REDUCING SURFACE REFLECTIONS USING POLARISING FILTER TECHNIQUES
}

\author{
N. Conen ${ }^{1 *}$, H. Hastedt ${ }^{1}$, O. Kahmen ${ }^{1}$, T. Luhmann ${ }^{1}$ \\ ${ }^{1}$ Jade University of Applied Sciences, Institute for Applied Photogrammetry and Geoinformatics (IAPG), Ofener Str. 16/19, \\ 26121 Oldenburg, Germany - (niklas.conen, heidi.hastedt, oliver.kahmen, luhmann)@jade-hs.de
}

\author{
Commission II, WG II/7
}

KEY WORDS: polarising filter, camera calibration, image matching, endoscopy, accuracy, completeness

\begin{abstract}
:
In dense stereo matching applications surface reflections may lead to incorrect measurements and blunders in the resulting point cloud. To overcome the problem of disturbing reflexions polarising filters can be mounted on the camera lens and light source. Reflections in the images can be suppressed by crossing the polarising direction of the filters leading to homogeneous illuminated images and better matching results.
\end{abstract}

However, the filter may influence the camera's orientation parameters as well as the measuring accuracy. To quantify these effects, a calibration and an accuracy analysis is conducted within a spatial test arrangement according to the German guideline VDI/VDE 2634.1 (2002) using a DSLR with and without polarising filter. In a second test, the interior orientation is analysed in more detail. The results do not show significant changes of the measuring accuracy in object space and only very small changes of the interior orientation $(\Delta \mathrm{c} \leq 4 \mu \mathrm{m})$ with the polarising filter in use.

Since in medical applications many tiny reflections are present and impede robust surface measurements, a prototypic trinocular endoscope is equipped with polarising technique. The interior and relative orientation is determined and analysed. The advantage of the polarising technique for medical image matching is shown in an experiment with a moistened pig kidney. The accuracy and completeness of the resulting point cloud can be improved clearly when using polarising filters. Furthermore, an accuracy analysis using a laser triangulation system is performed and the special reflection properties of metallic surfaces are presented.

\section{INTRODUCTION}

Correlation-based measurement procedures using two or more images usually require overlapping, well-structured and unambiguous image contents. One of the main challenges of such procedures is the matching of corresponding image points especially in scenarios where surface reflections occur.

The location of the reflection in the image depends on the spatial arrangement of the camera and light source. Reflections (bright hot spots) lead to uniform intensity distribution and thus may cause ambiguities for image matching. Furthermore, a reflection might be visible in one camera position, but invisible or less intense in another camera position. This unpredictable behaviour creates adverse conditions for image matching. Some applications that have to deal with reflections either segment, skip or interpolate these areas for dense image matching (Arnold et al., 2010).

For some purposes it might be useful to measure the surface including reflecting areas, for example in medical applications like surface reconstruction from endoscopic images or in industrial applications like three-dimensional inspection of welding seams or other shiny surfaces. Since surfaces in endoscopy are usually moist, the endoscopic light source causes many specular highlights. In addition, the surface is usually not stable. In comparison, welding seams are rigid. However, reflections are similar to endoscopic surfaces.

\subsection{Contribution}

In this contribution, an endoscopic measurement system is presented that can reliably deal with specular reflections on arbitrary surfaces. The system provides dense point clouds by semi-global matching using three images. Reflections on the surface are eliminated or suppressed using polarising filters for the camera lens and light source.

Since polarising filters add an optical component to the sensor system, the standard photogrammetric camera model (Brown, 1971) has to be validated. According to the German guideline VDI/VDE 2634.1 (2002) the maximum length measurement error $\left(\mathrm{LME}_{\max }\right)$ of a DSLR with and without polarising filter is determined and compared. Additionally, the interior orientation of the DSLR is evaluated in a further test field calibration under more stable conditions using a tripod and high quality lens. The interior and relative orientation of the trinocular endoscope is determined and analysed as well.

The effect of the polarising filter technique is demonstrated by observing a moistened pig kidney with different polarisation directions. The surface is reconstructed for each image triple representing one direction of polarisation. The crossed polarisation arrangement leads to the best reconstruction result since reflections are mostly suppressed. Furthermore, the special reflection behaviour of metallic surfaces is shown. In an application for surface reconstruction from laser profiles, polarising filters allow the concurrent segmentation of metallic surfaces, non-metallic surfaces and the projected laser line.

\footnotetext{
* Corresponding author
} 


\subsection{Light and Polarising Filters}

Light can be understood as a transversal electromagnetic wave that propagates in space along a propagation direction $\mathrm{z}$ (Figure 1a). The wavelength defines the colour and the amplitude defines the intensity. The direction of oscillation (Figure 1, red field vectors) describes the polarisation. In contrast to the wavelength and amplitude, the polarisation is not visible to humans.

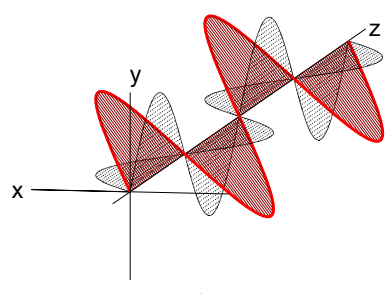

$\mathrm{a}$

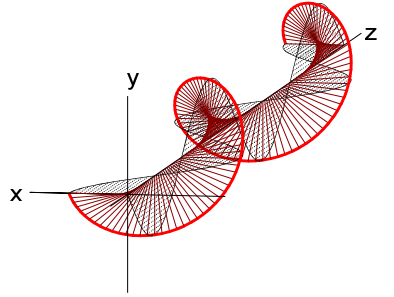

b
Figure 1. Light as a transversal wave with linear (a) and circular (b) polarisation.

The field vectors are perpendicular to the propagation direction and can rotate arbitrary. In case of linear polarisation, the field vectors form a plane (Figure 1a). In case of elliptic or circular polarisation, the field vectors rotate continuously around the propagation direction. The rotation can be described as phase shift between the horizontal and vertical projection of the wave (Figure 1b, black sinusoidal waves). Light is not polarised when the rotation is arbitrary and multiple polarisations are present at the same time.

Natural light sources (e.g. sun) and most artificial light sources (lightbulbs or LEDs) emit unpolarised light. A light beam becomes partly polarised when it is reflected, refracted, or scattered by a non-metallic object (dielectric material). If the angle of incidence corresponds to the Brewster angle, a complete polarisation arises perpendicular to the plane of incidence Jähne (2004).

When a (artificial) light source emits polarised light, the polarisation of a reflected beam only depends on the type of reflection, which may be directional or diffuse, depending on the roughness of the object. If the roughness is less than the wavelength of the incident light beam, the reflection is directional and diffuse vice versa. A diffuse reflection changes its polarisation, while it remains constant for a directional, i.e. specular, reflection. Hence, specular reflections from polarised light sources can be suppressed using polarising filters of perpendicular oscillation. If the directions of polarisation are parallel instead, specular reflections would become slightly more prominent whereas diffuse reflections are reduced. In general, two polarisation settings are known as cross and parallel polarisation. Both settings are illustrated in Figure 2. Further information on polarisation can be found in Jähne (2004).

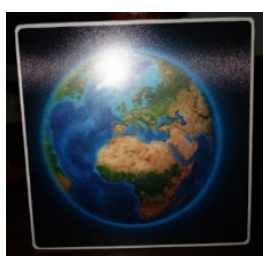

a

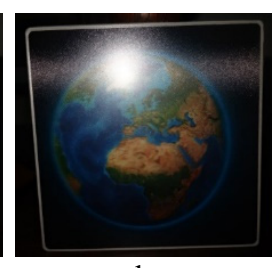

b

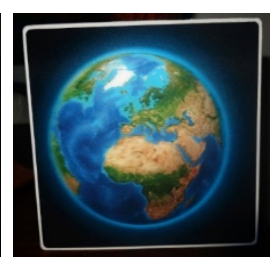

c
Figure 2. Experiment using polarised light source. Polarising filters are not in use (a), parallel (b) or crossed (c).

\subsection{Related Work}

Polarising filters are used in common and commercial photography and are particularly interesting for professional film and advertising industry. The filter allow capturing images with higher saturation and fewer reflections on shiny objects (Baumbach, 2014; Beitinger, 2016).

Kraus et al. (1992) and Menna et al. (2016) published photogrammetric applications of polarising filters to enhance contrast, increase colour saturation, achieve more homogeneous lightning conditions and, in particular, suppress specular reflections. Kraus et al. (1992) focused on the improvement in visual appearance of acquired images of the Behaim Globe; Menna et al. (2016) mainly used crossed polarising filters for reflection suppression and improved dense matching results for a cultural heritage object, the Etruscan Sarcophagus of the Spouses. A critical investigation of the influence on the interior parameters using polarising filters is not conducted.

Clancy et al. (2014) present an application for endoscopy. The authors simultaneously capture two images with crossed polarisation directions and segment the colour channels using a triple bandpass filter. Combining the polarisations and channels allow for a contrast-enhanced image in which very densely arranged and small blood vessels become visible. The proposed image enhancement may offer new possibilities in endoscopic diagnostics.

Atkinson et al. (2006) proposed a Shape-from-Polarisation (SfP) procedure to reconstruct a surface from images with three defined polarisation directions $\left(0^{\circ}, 45^{\circ}, 90^{\circ}\right)$. Herrera et al. (2013) applied this procedure to medical images acquired with a polarised endoscope. Experiments with different anatomical specimens show better results from SfP than from Shape-from-Shading.

The polarisation camera POLKA, distributed by the Fraunhofer Institute for Integrated Circuits (Fraunhofer, 2018), consists of an image sensor with a polarised microlens array. Each microlens has one of four defined polarisation directions $\left(0^{\circ}, 45^{\circ}, 90^{\circ}\right.$ and $\left.135^{\circ}\right)$ in order to generate four images with different polarisations from one observation. This allows an immediate analysis of the fibre direction of carbon fibre composites.

Another well-established application of polarising filters is the quality assessment of transparent dielectric (non-metallic) materials (like plastic utensils, e.g. plastic bottles) in photoelasticity. The stress distribution around discontinuities in those materials can be visualised when polarised light passes through an object and is captured with a cross-polarised camera system. The object transmission leads to birefringence that changes the polarisation direction and causes a colour spectrum in the captured image (Beyerer et al., 2012).

\section{CAMERA MODEL AND POLARISING FILTER}

Cross polarisation has not been used extensively in photogrammetry, although it is known for decades and wellestablished in some areas. An extensive validation of the photogrammetric measurement accuracy and an investigation of the influence on camera model parameters using polarising filters could not be identified within published research activities. This paper investigates the influence of polarising filters by experiments using professional DSLR, high quality lens, photogrammetric calibration and test procedures within a bundle adjustment. 


\subsection{Accuracy Evaluation}

According to the German guideline VDI/VDE 2634.1 (2002) the accuracy potential is determined for five different DSLR and flash setups with same NIKKOR 24mm-lens with and without polarising filter. The camera setups are
A Nikon D2X with an eccentric flash,
B Nikon D4 with an eccentric flash,
C Nikon D2X with a ring flash mounted on the lens,
D Nikon D4 with a ring flash mounted on the lens and
E Nikon D2X with a ring flash mounted on the tripod socket.

Setup $\mathrm{E}$ is added due to stability issues in setup $\mathrm{C}$ and $\mathrm{D}$. In setup $\mathrm{E}$, the gravity of the flash does not affect the lens geometry when rotating or tilting the camera, since the flash is separated from the lens. Figure 3 shows the different flash types.

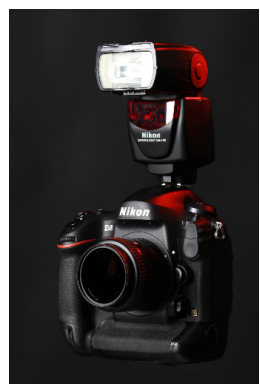

a

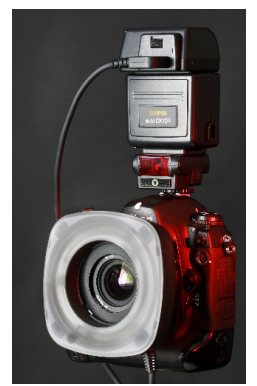

b

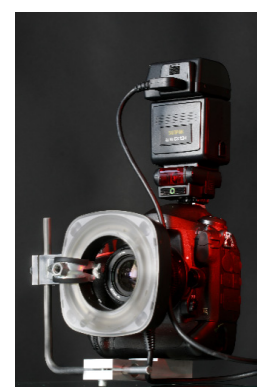

Figure 3. Exemplary camera setups. a) Nikon D4 with eccentric flash and polarising filter (B); b) Nikon D2X with ring flash mounted to the lens (C); c) Nikon D4 with ring flash mounted to the tripod socket $(\mathrm{E})$.

The accuracy evaluation is based on the determination of length measurement errors according to VDI/VDE 2634.1 (2002). Seven measuring lines, consisting of $\geq$ three retro-targets each, are arranged within a volume of about $2 \times 2 \times 1.5 \mathrm{~m}^{3}$ (Figure $4 \mathrm{a}$ ). In total, 58 calibrated distances are given as measurement artefacts between the retro-targets. The calibration accuracy of the distances is certified to less than $10 \mu \mathrm{m}$. As quality parameter, the length measurement error (LME) is determined as performance-target-deviation. The maximum absolute value of the length measurement errors is taken as $\mathrm{LME}_{\max }$ and as quality measure.

The volume is observed three times (test 1-3), each from 12 positions, in three height levels and with four different tilt angles. This results in a bundle of 144 images (Figure $4 b$ ).

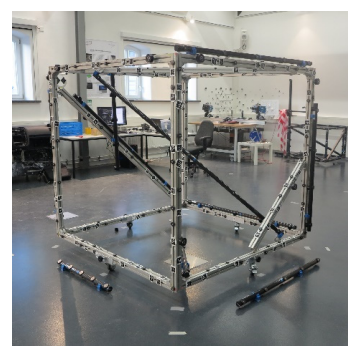

a

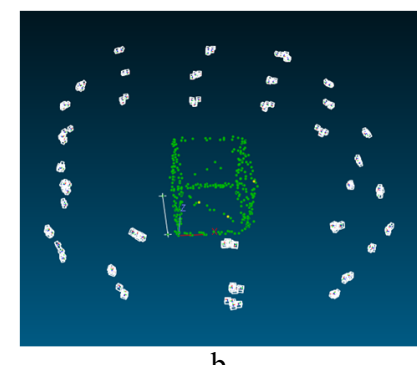

$\mathrm{b}$
Figure 4. VDI test volume (a) and imaging setup (b).

A bundle adjustment for each dataset results in an RMS of object point precision in each axis of about $20 \mu \mathrm{m}$. This corresponds to the expected quality level of the used camera setup. Three calibrated lengths along the coordinate axes are applied as scale constraints to the bundle adjustment. The measured distances for the calibrated length are determined from the resulting object point coordinates. The largest absolute deviation between the 58 calibrated and measured lengths (LMEmax) for each camera setup are summarised in Table 1 and Figure 5. The LMEmax provides information about the measuring accuracy of each setup.

\begin{tabular}{|l|c|c|c|}
\hline \multirow{2}{*}{ Setup/Pol.filter } & \multicolumn{3}{|c|}{ LME $_{\max }[\mathrm{mm}]$} \\
& Test 1 & Test 2 & Test 3 \\
\hline A/pol & 0.075 & 0.110 & 0.158 \\
A & 0.086 & 0.134 & 0.110 \\
B/pol & 0.117 & 0.069 & 0.134 \\
B & 0.101 & 0.087 & 0.132 \\
C/pol & 0.223 & 0.227 & 0.121 \\
C & 0.157 & 0.183 & 0.135 \\
D/pol & 0.135 & 0.124 & 0.198 \\
D & 0.219 & 0.174 & 0.205 \\
E/pol & 0.060 & 0.091 & 0.098 \\
E & 0.070 & 0.071 & 0.077 \\
\hline
\end{tabular}

Table 1. Resulting $\mathrm{LME}_{\max }$ for camera setups.

\section{$\mathrm{LME}_{\max }$ for different camera setups}

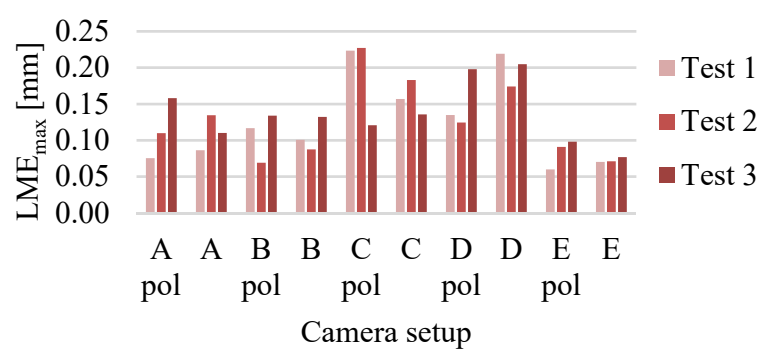

Figure 5. Diagram of LMEmax for different camera setups.

The diagram reveals three setup groups of similar LMEmax. The $\mathrm{LME}_{\max }$ of the setups A-B, C-D and E are similar, however, the $\mathrm{LME}_{\max }$ between the groups are quite different. These differences are caused by the flash setup. The gravity of the ring flash mounted to the lens (group C-D) influences the lens geometry when tilting the camera. Thus, camera parameters are not stable and LME are larger. The groups A-B and E are captured with a flash separated from the lens and show better results. However, group A-B shows a marginally decrease in accuracy compared to E. That may be caused by an eccentric image measurement of the retro-target for A-B. The differences in the tests are caused by the NIKKOR $24 \mathrm{~mm}$-lens which mechanical geometry changes slightly during observation. This influences the calibration stability and thus the LME.

The polarising filter has no significant effect on the LME. The LME of same setup with and without filter are quite similar. For that reason, polarising filters are applicable to a standard photogrammetric camera model and bundle adjustment with respect to the investigated setups.

\subsection{Analysis of Interior Camera Parameters}

The interior orientation is determined simultaneously by the bundle adjustments from the VDI datasets for each camera setup. In these experiments, no significant changes in the interior orientation have been detected. However, the comparability of the results is limited due to the instability of the camera and lens geometry and remaining measurement uncertainties. In order to allow for a reliable comparison of the interior orientation parameters, whether using the polarising filter or not, a second assessment is evaluated. 
Therefore, an additional test-field calibration using the Nikon D4 with a $35 \mathrm{~mm}$-ZEISS-lens (being of higher quality than the $24 \mathrm{~mm}-\mathrm{NIKKOR}-\mathrm{lens}$ ) and a tripod is conducted. This setup offers a higher stability of the interior camera geometry during the tests while moving the test-field instead. The eccentric flash is used for image acquisition in order to enable cross-polarisation. The cross-polarisation setup is achieved by a polarising film pasted onto the flash and a polarising filter mounted to the lens in crossed polarisation direction (Figure 6a). Six tests are performed, four with and two without polarising filters. Thereby, a shiny carbon test field with standard targets is observed from about 34 different positions by moving the test-field. An exemplary image without (b) and with (c) crossed polarising filters is shown in Figure 6.

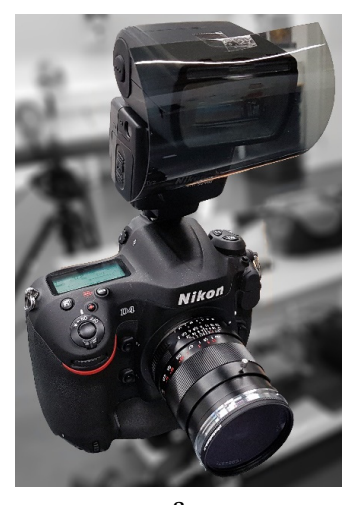

a

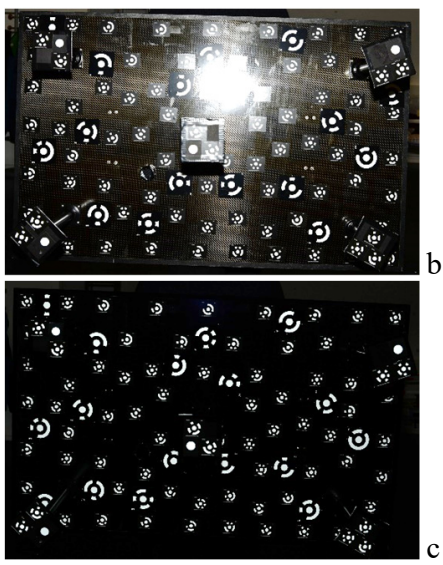

Figure 6. Nikon D4 with $35 \mathrm{~mm}$-ZEISS-lens with polarising filter and eccentric flash with polarising film (a); carbon test field captured without (b) and with (c) crossed polarising filters.

The interior orientation parameters for each dataset are computed by a bundle adjustment. The obtained parameters are the principal distance $(\mathrm{c})$, principal point $\mathrm{H}\left(\mathrm{x}^{\prime} 0, \mathrm{y}^{\prime}\right)$, radial-symmetric lens distortion (A1, A2, A3), decentring distortion (B1, B2), affinity and shear (C1, C2) (Luhmann et al., 2014). The parameters are determined significantly with a significance level of $95 \%$ for each dataset, except for parameter $\mathrm{C} 2$ in one test. The resulting parameters for an exemplary test with (w pol.filter) and without (w/o pol.filter) polarising filters is shown in Table 2.

The influence of the polarising filter is indicated by the deviation $(\Delta)$ of the parameters determined with and without polarising filter. Its standard deviations $\left(\mathrm{s}_{\Delta}\right)$ are propagated in order to test its significance. The mean deviation $(\varnothing \Delta)$ and corresponding standard deviations of the mean ( $\mathrm{s} \varnothing \Delta)$ are listed in Table 2 . The mean differences are determined with a significance level of $95 \%$, except of parameter $\mathrm{C} 2$.

The comparison shows the influence on each parameter caused by the polarising filter. The most prominent parameters are the principal distance and the principal point. The principal distance is $4 \mu \mathrm{m}$ longer in average when polarising filters are applied. Additionally, the principal point is slightly shifted to the image centre. The shift equals $0.7 \mathrm{px}$ in $\mathrm{x}$ - and $1.6 \mathrm{px}$ in $\mathrm{y}$-direction with a pixel size of $7.3 \mu \mathrm{m}$. These changes are reliable due to refraction of the additional glass layer in front of the lens. Furthermore, the behaviour of the principal distance and principal point seem to be correlated. As the principal distance gets longer, the principal point is shifted to the image centre. Another reason for the deviations might be due to a tilt of the filter with respect to the image sensor, which may be different for each camera and lens combination because of limited manufacturing accuracy.

\begin{tabular}{|c|c|c|c|c|}
\hline$[\mathrm{mm}]$ & \multicolumn{2}{|c|}{$\begin{array}{l}\text { determined parameters } \\
\text { w/o, w pol.filter }\end{array}$} & $\begin{array}{c}\text { mean } \\
\text { deviation } \\
\varnothing \Delta\end{array}$ & $\begin{array}{c}\text { std. dev. } \\
\text { of mean } \\
\text { s } \varnothing \Delta\end{array}$ \\
\hline $\mathrm{c}$ & -35.988 & -35.991 & 0.004 & 0.001 \\
\hline $\mathrm{x}_{0}^{\prime}$ & 0.0 & 0.0 & 0.005 & 0.001 \\
\hline $\mathrm{y}_{0}^{\prime}$ & 0.135 & 0.1 & 0.013 & 0.005 \\
\hline A1 & $-6.80 \mathrm{E}-05$ & $-6.94 \mathrm{E}-05$ & $1.13 \mathrm{E}-06$ & $2.39 \mathrm{E}-07$ \\
\hline A2 & $3.81 \mathrm{E}$ & 4.47E-08 & $-5.08 \mathrm{E}-09$ & $1.23 \mathrm{E}-09$ \\
\hline A3 & 1. & $15 \mathrm{E}-12$ & 5.9 & $1.73 \mathrm{E}-12$ \\
\hline B1 & 2.88 & $E-06$ & 6 & IE-07 \\
\hline B2 & 5.34 & $5 \mathrm{E}-06$ & 1.8 & $6.61 \mathrm{E}-07$ \\
\hline $\mathrm{C} 1$ & $8.48 \mathrm{E}$ & $0 \mathrm{E}-05$ & $1.59 \mathrm{E}-05$ & $4.59 \mathrm{E}-06$ \\
\hline $\mathrm{C} 2$ & $-3.78 \mathrm{E}-05$ & $-6.26 \mathrm{E}-06$ & $2.13 \mathrm{E}-05$ & $3.88 \mathrm{E}-05$ \\
\hline
\end{tabular}

Table 2. Exemplary interior orientation parameters with and without polarising filters; mean over all deviation and its standard deviation.

The radial-symmetric lens distortion curves of the exemplary parameter sets are visualised in Figure 7. The deviation in the distortion curves at the image border is only $0.3 \mathrm{px}$. The influence to radial-symmetric lens distortion as well as decentring distortion, affinity and shear is very low but present.

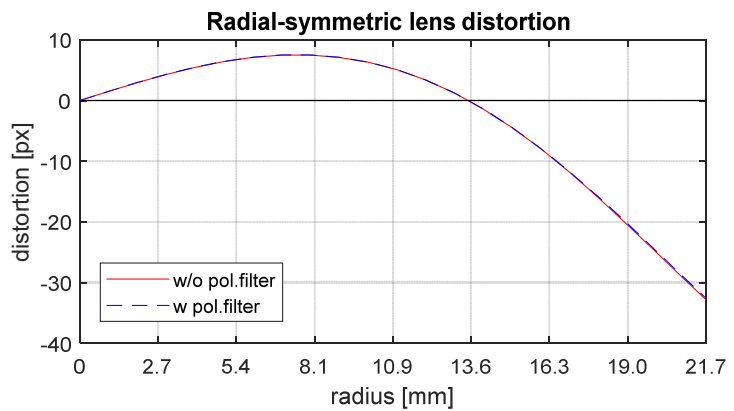

Figure 7. Radial-symmetric lens distortion curve of Nikon D4 with $35 \mathrm{~mm}$-ZEISS-lens with and without polarising filter.

This experiment shows that calibration parameters, especially the principal distance and principal point, vary slightly when polarising filters are used. To enable high accuracy measurements, it is appropriate to calibrate the camera system for each setup individually. Furthermore, the parameters of a test should always be considered as a unique set since parameters correlate with each other.

\subsection{Orientation of Trinocular Endoscope}

According to the results of the previous subsections, it is assumed that other camera systems with polarising filters and similar imaging properties can also be described by the standard photogrammetric camera model. Hence, a special trinocular camera system equipped with a ring flash and a polarising filter (Figure $8 \mathrm{~b}$ ) is evaluated regarding the determination of orientation parameters and dense image matching processing. The prototypical system consists of three equilateral arranged cameras with a diameter of $6 \mathrm{~mm}$ each (Figure 8a). The outer diameter of the three cameras is about $14 \mathrm{~mm}$. The image resolution is $640 \times 640 \mathrm{px}^{2}$ with a pixel size of $3.6 \mu \mathrm{m}$. The system resembles a trinocular endoscope that allows stereovision and reliable 3D measurements in surgical scenarios (Conen et al., 2017). Due to size restrictions the height-to-base ratio is approximately 10:1. 

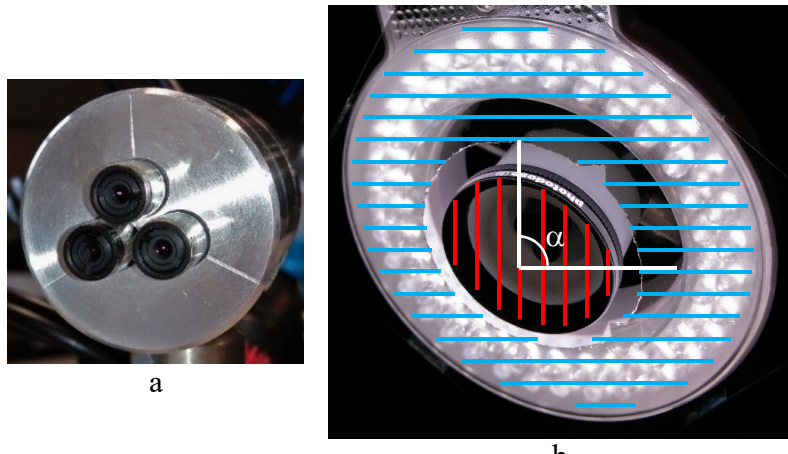

Figure 8. Trinocular miniature camera system (a) and system setup with light source and filter (b); direction of polarisation is indicated as blue and red lines, angle of polarisation $\alpha$ is $90^{\circ}$ (example cross-polarisation).

The interior orientation as well as the relative pose between the three cameras (relative orientation) are determined with and without cross-polarisation setup. The relative orientation is defined by translation parameters $(\mathrm{X}, \mathrm{Y}, \mathrm{Z})$ and rotation angles $(\omega, \varphi, \kappa)$. The left camera defines the origin of the local camera coordinate system $\left(X_{0}=Y_{0}=Z_{0}=\omega_{0}=\varphi_{0}=\kappa_{0}=0\right)$. As in the previous section, the interior orientation parameters are determined by a bundle adjustment using a three dimensional test-field. The relative orientation is estimated by multiple resections with fixed interior parameters achieved from a previous bundle adjustment. The results are shown in Table 3 and the radial-symmetric lens distortion curves are visualised in Figure 9.

\begin{tabular}{|c|r|r|r|}
\hline$[\mathrm{mm}]$ & \multicolumn{2}{|c|}{$\begin{array}{r}\text { determined parameters } \\
\text { w/o, w pol.filter }\end{array}$} & deviation \\
\hline $\mathrm{rad}]$ & \multicolumn{2}{|c|}{ without } & \multicolumn{1}{c|}{$\Delta$} \\
\hline $\mathrm{c}_{0}$ & -6.036 & -6.043 & 0.006 \\
$\mathrm{X}_{0.0}$ & -0.015 & -0.013 & -0.002 \\
$\mathrm{y}_{0.0}^{\prime}$ & -0.165 & -0.164 & -0.002 \\
\hline $\mathrm{c}_{1}$ & -5.975 & -5.974 & 0.000 \\
$\mathrm{X}_{0.1}^{\prime}$ & 0.074 & 0.060 & 0.014 \\
$\mathrm{y}_{0.1}^{\prime}$ & -0.138 & -0.137 & -0.002 \\
\hline $\mathrm{c}_{2}$ & -5.983 & -5.985 & 0.001 \\
$\mathrm{x}_{0.2}$ & 0.008 & 0.000 & 0.007 \\
$\mathrm{y}_{0.2}^{\prime}$ & 0.032 & 0.031 & 0.001 \\
\hline $\mathrm{X}_{1}$ & 7.691 & 7.692 & -0.001 \\
$\mathrm{Y}_{1}$ & -0.657 & -0.635 & -0.022 \\
$\mathrm{Z}_{1}$ & 0.196 & 0.076 & 0.121 \\
$\omega_{1}$ & $-1.31 \mathrm{E}-06$ & $-1.06 \mathrm{E}-03$ & $1.06 \mathrm{E}-03$ \\
$\varphi_{1}$ & $-5.40 \mathrm{E}-03$ & $-3.26 \mathrm{E}-03$ & $-2.14 \mathrm{E}-03$ \\
$\kappa_{1}$ & $1.64 \mathrm{E}-02$ & $1.47 \mathrm{E}-02$ & $1.72 \mathrm{E}-03$ \\
\hline $\mathrm{X}_{2}$ & 4.517 & 4.527 & -0.010 \\
$\mathrm{Y}_{2}$ & 5.360 & 5.358 & 0.002 \\
$\mathrm{Z}_{2}$ & -0.052 & -0.115 & 0.064 \\
$\omega_{2}$ & $8.07 \mathrm{E}-03$ & $7.59 \mathrm{E}-03$ & $4.76 \mathrm{E}-04$ \\
$\varphi_{2}$ & $-2.26 \mathrm{E}-03$ & $-4.07 \mathrm{E}-04$ & $-1.86 \mathrm{E}-03$ \\
$\kappa_{2}$ & $-2.82 \mathrm{E}-03$ & $-3.61 \mathrm{E}-03$ & $7.97 \mathrm{E}-04$ \\
\hline
\end{tabular}

Table 3. Interior and relative orientation parameters with and without polarising filters and their deviations; the deviations are calculated with exact values and rounded.

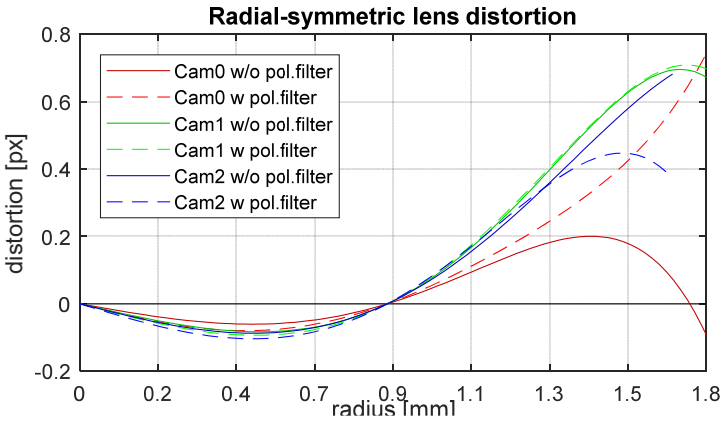

Figure 9. Radial-symmetric lens distortion curves of miniature cameras with and without polarising filter.

The results for the interior orientation show a similar but weaker trend in parameters as in the Nikon D4 test. In comparison to the camera size, the deviations are still in a similar range of values. The principal distances are slightly longer $(6 \mu \mathrm{m})$ or do not change significantly with polarising filter. The principal points are slightly shifted $(0.7-4.0 \mathrm{px})$ to the image centres. The radialsymmetric lens distortion is relatively low. Hence, the influence of the polarising filter is mainly less than $0.2 \mathrm{px}$ (Figure 9).

The results for the relative orientations of the right and top camera to the left show larger changes in $\mathrm{Z}$ direction than in $\mathrm{XY}$ direction. The projection centres of the right and top camera are located approximately $0.1 \mathrm{~mm}$ farther from the object than the left camera. Due to correlations between the $\mathrm{Z}$ direction and the principal distance, the shift in $\mathrm{Z}$ position may also be caused by refraction of the filter. The shift in XY position may be caused by a tilt between the filter and image sensor.

Due to a limited stability of the interior camera, the lens geometry and miniaturisation issues, the determination of orientation parameters for an endoscopic system is not as reliable as for a DSLR. For practical applications a standardised and fast calibration procedure is recommended. The resulting orientation parameters for cross-polarisation setup are used in the following sections for further image analysis.

\section{EXPERIMENTS AND RESULTS}

As described in the introduction, reflections can make stereoscopic image matching quite difficult. To improve matching results for applications having poor illumination conditions, a trinocular system which suppresses disturbing reflections is introduced. As described in Conen et al. (2016), a dense point cloud from the observed surface is computed by a semi-global matching approach for three images. The presented trinocular system and the matching approach from Conen et al. (2016) is used to evaluate the effect of polarising filters on image matching. Therefore, two experiments on a moistened pig kidney are conducted (Figure 10). The first experiment evaluates matching results from images with different degrees of reflexion suppression. The second experiment compares a matching result from a polarised endoscope with a result from a laser triangulation sensor of higher accuracy. In an additional third experiment, a prototypical laser triangulation sensor is equipped with polarising filters to demonstrate the properties of different materials regarding polarisation. The polarising filter allows for segmentation of metallic surfaces, laser lines and photogrammetric targets in parallel. 


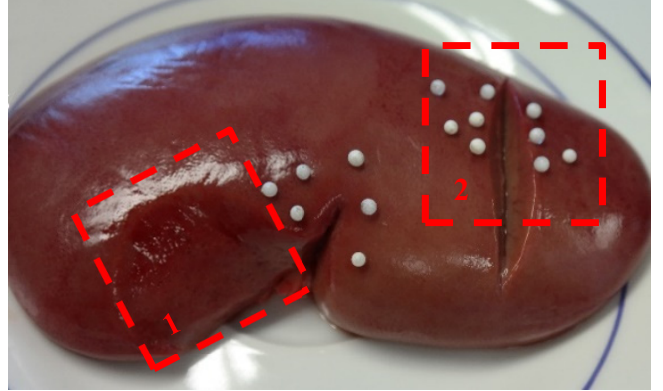

Figure 10. Specimen of a moistened pig kidney with highlighted areas of investigation for experiments.

\subsection{Reflexion Suppression for Image Matching}

In the first experiment, an area of $2 \times 2 \mathrm{~cm}^{2}$ of a moistened pig kidney (Figure 10, area 1) is observed from one fixed position with five different angles of polarisation: $0^{\circ}$ (parallelpolarisation), $22.5^{\circ}, 45^{\circ}, 67.5^{\circ}$ and $90^{\circ}$ (cross-polarisation). An additional measurement with cross-polarisation setup is conducted to check the precision of the 3D reconstruction process. An ex-vivo specimen of a kidney is chosen to simulate the object properties during a laparoscopic partial nephrectomy (i.e. resection of a tumour on a kidney using endoscopes for abdominal or pelvic cavities).

A dense matching with identical parameters is performed on each image triple. Since the dataset acquired using cross-polarisation $\left(90^{\circ}\right)$ consists of no specular reflections and lead to the most complete and homogeneous result, its mesh is used as reference for 3D comparison (cloud-to-mesh). The comparison to the second cross-polarisation setup leads to a standard deviation of shortest distances of $105 \mu \mathrm{m}$. Since a stable absolute orientation is assumed, the standard deviation represents the precision. A Monte-Carlo simulation performed in Conen et al. (2017) leads to a standard deviation for the depth of $247 \mu \mathrm{m}$ for a similar imaging distance $(75 \mathrm{~mm})$ and an assumed measurement accuracy in image space of half a pixel. However, Conen et al. (2017) mentioned that due to semi-global optimisation and subpixel interpolation a more optimistic measurement accuracy would be more realistic. A quarter pixel accuracy results in a standard deviation for the depth of about $124 \mu \mathrm{m}$, which is comparable to the derived precision of the second crosspolarisation measurement. Therefore, one quarter of a pixel is considered as measurement accuracy in image space for this experiment.

The results of the 3D comparisons between different angles of polarisation are shown in Figure 11. The fully saturated red and blue spots in the 3D comparison show approximately five times higher deviations compared to the precision of the $3 \mathrm{D}$ reconstruction process.

As expected, the amount and intensity of specular reflections grow with a decreased angle of polarisation. The reflections lead to blunders that partly deviate more than $\pm 0.5 \mathrm{~mm}$ from the reference. The standard deviations increase with smaller angles of polarisation. In case of the parallel polarisation, the fully saturated regions seem to be smoothed by semi-global matching, whereby the standard deviation becomes small. In conclusion, the standard deviations indicate a loss in accuracy with higher reflections.

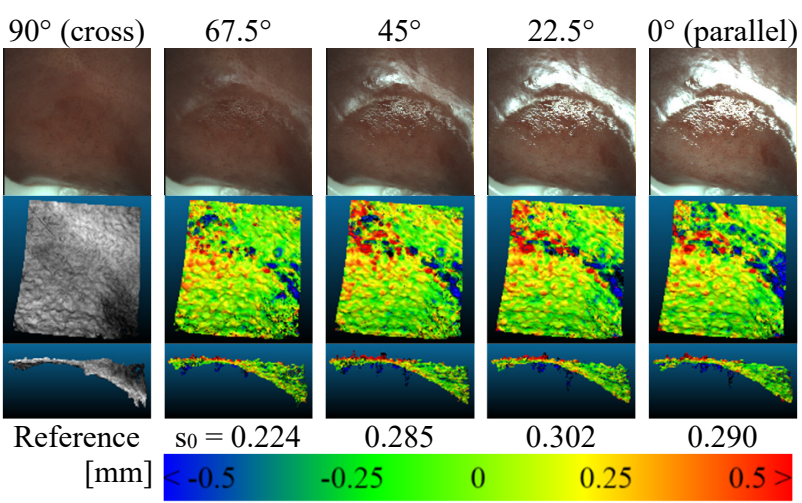

Figure 11. Comparison of different angles of polarisation. Top: original left image; middle: point cloud top view; bottom: point cloud side view; $90^{\circ}$ : reference in greyscale; other angles: $3 \mathrm{D}$ deviations w.r.t. $90^{\circ}$ in colour-code.

\subsection{Comparison with Laser Triangulation Sensor}

An accuracy assessment of the trinocular system equipped with crossed polarising filters is conducted practically by a comparison with measurements from a laser triangulation sensor (KEYENCE LJ-V7080). According to the manufacturer, the repeatability is $0.5 \mu \mathrm{m}$ in depth and $10 \mu \mathrm{m}$ in direction of the laser line. An incision of approximately $5 \mathrm{~mm}$ depth of the kidney (Figure 10, area 2) is captured with a XY resolution in object space of about $50 \times 100 \mu \mathrm{m}^{2}$. The trinocular system captures the same area with an imaging distance of about $75-80 \mathrm{~mm}$ and a resulting resolution in object space of about $50 \times 50 \mu \mathrm{m}^{2}$. The different resolutions are compensated by meshing the point cloud of the laser triangulation sensor.

The datasets are initially registered using spherical beads of about $2.25 \mathrm{~mm}$ in diameter. In case of the laser triangulation sensor, the centres of the beads are determined by best fitting spheres in the point cloud (Figure 12a) as it is common for laser scanning purposes. In case of the trinocular camera system, the centres are determined by measuring ellipses in image space (Figure 12b) and applying forward intersection as it is common in photogrammetry.

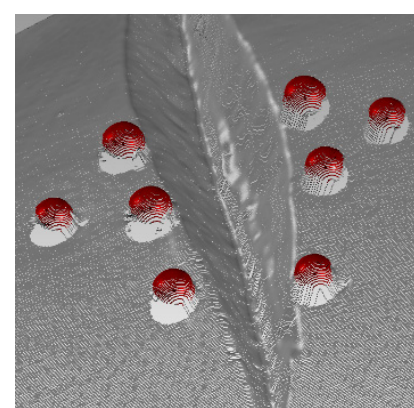

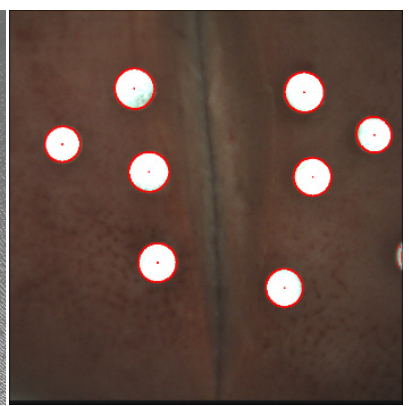

b
Figure 12. Target point detection; (a) point cloud and selected spherical points (red) of laser triangulation sensor; (b) measured ellipses in one image of the trinocular system.

The registration leads to a 3D RMS of $0.059 \mathrm{~mm}$ using eight target points. In order to just compare the surface points of the kidney the beads are deleted from the point clouds. The 3D comparison leads to a mean offset of $-0.164 \mathrm{~mm}$ and a standard deviation of distances of $0.215 \mathrm{~mm}$. In order to analyse the 
remaining offset a fine registration using iterative-closest-point (ICP) algorithm is applied. The ICP minimises the mean offset to $-0.013 \mathrm{~mm}$ with a standard deviation of $0.203 \mathrm{~mm}$. The standard deviation indicates the precision of the experiment. To define the absolute measurement accuracy of the trinocular system, the registration has to be considered. As shown in the results of the registration using beads an offset is detected. The offset is influenced by different 3D point measurement techniques and not perfectly spherical beads. For this experiment the absolute accuracy results to $-0.164 \mathrm{~mm}$. Referring to the Monte-Carlo simulation that equals a measurement accuracy in image space of about half a pixel.

The deviations after ICP are visualised in Figure 13 as colourcoded point cloud. The largest deviations occur at the deepest point of the incision due to a limited resolution and a smoothing effect caused by the matching. The flanks of the incision as well as the surrounding surface consists of lower deviations.

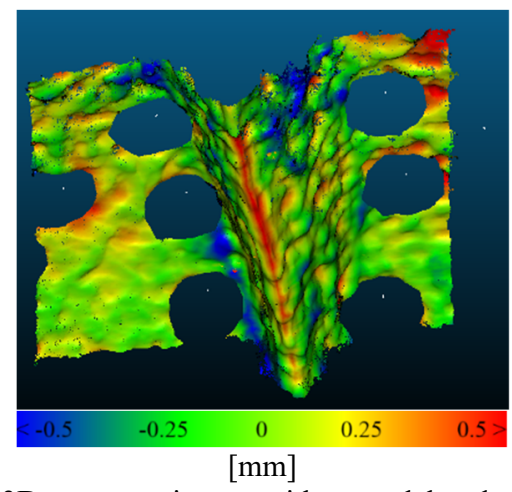

Figure 13. 3D comparison with model derived from triangulation sensor.

Without crossed polarising filters shiny or reflective areas would probably be inaccurate or not measured at all. With respect to the Monte-Carlo simulation, previous experiments from Conen et al. (2017) and the results of this paper, the resulting standard deviations are considered reliable. Even though polarising filters add an optical component to the camera model, their impact in accuracy is marginal. However, if there are reflections present in the scene, polarising filters offer a great advantage in accuracy, reliability and completeness.

\subsection{Segmentation of Metallic Materials}

The reflection and polarisation properties of metallic surfaces are different to those of non-metallic surfaces. Any reflection, whether diffuse or directed, does not change its polarisation properties. Hence, polarised light reflected on a metallic surface is completely filtered by a crossed polarising filter resulting in almost black pixels in the image. This property allows the distinction between metallic and non-metallic objects.

A practical application of this property might be a measurement of welding seams using a handheld laser triangulation sensor. The measured profiles of the triangulation sensor are oriented by paper targets attached to the object. These targets define a local coordinate system in which the oriented profiles form a surface of the welding seam. However, measuring the laser line and the paper targets simultaneously in one image is quite challenging due to different lightning requirements; the laser line requires short exposure times (Figure 14a) whereas the paper targets rely on longer exposure times and additional light sources. With a compromising lightning setting many ambiguities for profile measurement occur due to reflections on the welding seam
(Figure 14b). In addition, the reflections are discontinuous due to the arbitrary wave-like microstructure of the welding seam. Thus, reflections vary, even when camera position change slightly.

Crossed polarising filters can overcome this problem. Since the polarised light reflected by the welding seam does not pass the crossed polarising filter, only the reflection of the laser line and paper targets are visible in the image (Figure 14c). Although laser is already polarised, its reflection passes the filter due to a different direction of polarisation. The filter slightly reduces its intensity, however, it is still enough for reliable image measurement.

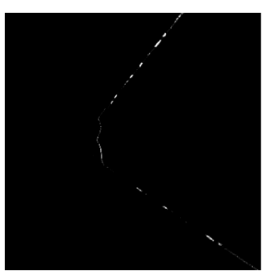

a

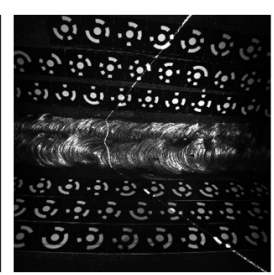

b

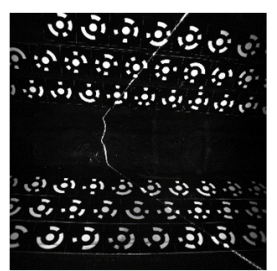

c
Figure 14. Acquired images of a welding seam with laser line and photogrammetric paper targets; (a) short exposure time; (b) compromising exposure time; (c) crossed polarising filter.

The last experiment demonstrates the special polarisation properties of metallic surfaces and gives an example for a useful application. The polarising technique might be a solution for many applications in which disturbing reflections occur. Despite the technique is well known in some areas there is still potential for photogrammetric purposes.

\section{DISCUSSION AND CONCLUSION}

In this paper it could be shown that polarising filters can be used with a standard photogrammetric camera model and bundle adjustment. The effect of polarising filters is negligible in the achieved range of accuracy with respect to target measurements in a VDI test environment. An experiment with a more stable camera setting shows the variations of the interior camera parameters when polarising filters are used. The filter mainly influences the principal distance and the principal point. However, the common photogrammetric camera model can still describe these variations. Consequently, it is assumed that polarising filters are applicable to other camera systems with similar imaging properties like the presented trinocular endoscope. The influence in $\mathrm{Z}$ direction of the relative orientation is noticeable large. However, it is also highly correlated to the principal distance.

The effect of cross-polarisation on moistened organic surfaces is impressive and enables a polarised system to measure in more complicated scenarios. A significant drawback is the loss of intensity, which requires a more powerful illumination. The advantage on the other side is a very homogeneous appearance of the moistened surface since only diffuse reflections are captured. Only the texture of the object is visible and no disturbing reflections occur.

An accuracy assessment is conducted on a pig kidney with an incision using a laser triangulation sensor and the polarised endoscopic system. After ICP the standard deviation of the shortest distances between the datasets (cloud-to-mesh) is determined to be $0.203 \mathrm{~mm}$. Considering the height-to-base ratio of about 10:1, the Monte-Carlo simulation and tests from Conen et al. (2017), the achieved accuracy is plausible. For laparoscopic surgeries, which are operations in the abdominal or pelvic 
cavities, the achieved accuracy is sufficient. However, a real-time computation and stitching of point clouds over time to access larger areas is not implemented yet. Not only matching algorithms profit from polarising filters. Another benefit is a better and cleaner vision to the surgeon, which probably makes an operation less demanding to the surgeon's eyes.

A measurement of a welding seam using a laser triangulation sensor demonstrates a useful application of polarising filters for non-medical purposes. The special reflection properties of metallic surfaces enable a simultaneous measurement of the laser line and photogrammetric paper targets for orientation of profiles in only one image. This experiment shows a great potential for other applications that have to deal with disturbing reflections.

Complex reflecting surfaces of various materials can be measured by conventional image matching or laser triangulation techniques when polarising filters are applied smartly. The filters can be used with standard photogrammetric camera model and eliminate disturbing reflections reliable.

\section{ACKNOWLEDGEMENT}

This project is funded alias OrthoScan by the European Regional Development Fund and the federal state of Lower Saxony.

\section{REFERENCES}

Atkinson, G. A. \& Hancock, E. R., 2006. Recovery of surface orientation from diffuse polarization. In: IEEE Transactions on Image Processing, Vol. 15, No. 6, pp. 1653-1664. doi: 10.1109/TIP.2006.871114.

Arnold, M., Ghosh, A., Ameling, S. \& Lacey, G., 2010. Automatic Segmentation and Inpainting of Specular Highlights for Endoscopic Imaging. In: EURASIP Journal on Image and Video Processing, 2010: 814319, doi: 10.1155/2010/814319.

Baumbach, D., 2014: Die Kreuzpol Blitztechnik. fotografenwelt, TB Foto Team, http://www.fotografenwelt.de/fototipps/fototipps-kreuzpolblitzen-eine-professionelleblitztechnik/ (09.02.2018).

Beitinger, A., 2016. Polarisationsfilter. fotovideotec, $\mathrm{http}$ ://fotovideotec.de/polfilter/ (09.02.2018).

Brown, D.C., 1971. Close-Range Camera Calibration. In: Photogrammetric Engineering, pp. 855-866.

Clancy, N. T., Arya, S., Qi, J., Stoyanov, D., Hanna, G. B., \& Elson, D. S., 2014. Polarised stereo endoscope and narrowband detection for minimal access surgery. In: Biomedical Optics Express, 5(12), pp. 4108-4117. doi: 10.1364/BOE.5.004108.

Conen, N., Jepping, C., Luhmann, T. \& Maas, H.-G., 2016. Rectification and robust matching using oriented image triplets for minimally invasive surgery. In: ISPRS international annals of the photogrammetry, remote sensing and spatial, Inf Sci 3(3), pp. 27-34.

Conen, N., Luhmann, T. \& Maas, H.-G., 2017. Development and Evaluation of a Miniature Trinocular Camera System for Surgical Measurement Applications. In: Journal of Photogrammetry, Remote Sensing and Geoinformation Science, Vol. 85, Issue 2, pp. 127-138.
Fraunhofer 2018. POLKA. Fraunhofer IIS, https://www.iis.fraunhofer.de/de/ff/sse/ims/tech/polarisationska mera.html, Patent: http://publica.fraunhofer.de/dokumente/N311094.html (09.02.2018).

Herrera, S.E.M., Malti, A., Morel, O. \& Bartoli, A., 2013. Shapefrom-polarization in laparoscopy. In: IEEE 10th International Symposium on Biomedical Imaging, pp. 1412-1415.

Jähne, B., 2004. Practical Handbook on Image Processing for Scientific and Technical Applications. CRC Press, 2nd Ed.

Kraus, K., Tschannerl, J., Halmer, A. \& Rottensteiner, F., 1992. Working on the Behaim Globe using Photogrammetric Methods. In: Journal for the Study of Globes and Related Instruments, 40/41, pp. 9-19.

Luhmann, T., Robson, S., Kyle, S. \& Boehm, J., 2014. CloseRange Photogrammetry and 3D Imaging. De Gruyter textbook, 2nd edition, ISBN 978-3-11-030269-1.

Menna, F., Nocerino, E., Remondino, F., Dellepiane, M., Callieri, M. \& Scopigno, R., 2016. 3D Digitization of an Heritage Masterpiece - a Critical Analysis on Quality Assessment. In: The International Archives of the Photogrammetry, Remote Sensing and Spatial Information Sciences, 41, pp. 675-683.

VDI/VDE 2634.1 (2002): Optical 3-D measuring systems Imaging systems with point-by-point probing. $V D I$, Düsseldorf. 